\title{
INFLUÊNCIA DA IRRIGAÇÃO SOBRE O ALBEDO
}

\author{
Mário de Miranda V.B.R. Leitão ${ }^{1}$ \& Gertrudes Macario de Oliveira ${ }^{2}$
}

\begin{abstract}
RESUMO
Uma análise dos efeitos e da influência da irrigação sobre o albedo de uma cultura de amendoim, ao longo do ciclo de desenvolvimento e, em particular, durante um dia em que houve irrigação, é realizada neste estudo, cuja pesquisa de campo foi desenvolvida no perímetro irrigado da Estação Experimental da CODEVASF, no município de Rodelas, BA, no período de setembro a dezembro de 1996. Os resultados mostraram que o albedo variou significativamente nos dias em que foram efetuadas irrigações e nos dias imediatamente posteriores. Sob condições normais, o albedo variou de um máximo de $29,8 \%$ na fase inicial do experimento, com o solo ainda descoberto, a um mínimo de $18,6 \%$ no final da fase de desenvolvimento de vagem; contudo, nos dias de irrigação, dependendo da hora em que esta foi efetuada, houve influência diferenciada no valor do albedo médio diário, que chegou a reduzi-lo em até $6,4 \%$, quando as irrigações ocorreram nas primeiras horas da manhã.
\end{abstract}

Palavras-chave: balanço de radiação, irrigação, amendoim

\section{INFLUENCE OF IRRIGATION ON ALBEDO}

\begin{abstract}
The influence of irrigation on the albedo of a peanut crop during its development cycle, and in particular on the day when irrigation took place, is analyzed in this study. The field research was conducted in the irrigated perimeter of the Experimental Station of CODEVASF, in the municipal district of Rodelas, BA, during the period of September to December of 1996. The results showed that the albedo suffered significant variations on the days of irrigation as well as on the day after irrigation. Under normal conditions, the albedo varied from a maximum of $29.8 \%$ in the initial phase of the experiment with the soil still uncovered, to a minimum of $18.6 \%$ at the end of the kernel development phase. However, on the days of irrigation, the albedo varied with the time of irrigation. The daily mean value of the albedo reduced by $6.4 \%$ when the irrigations took place in the early hours of the morning.
\end{abstract}

Key words: radiation balance, irrigation, peanut

Recebido em 09/03/2000, Protocolo 024/00

${ }^{1}$ Prof. Dr. em Meteorologia do Departamento de Ciências Atmosféricas - CCT/UFPB. CP 10.099, CEP 58109 - 970, Campina

Grande, PB. Tele/Fax: (0xx83) 310 1202, Ramal 32.E-mail: miranda@dca.ufpb.br

${ }^{2}$ Doutoranda do Curso de Recursos Naturais - CCT/UFPB. E-mail: gertrude@dca.ufpb.br 


\section{INTRODUÇÃO}

A radiação solar que atinge a superfície da Terra, é um parâmetro de fundamental importância para os diversos processos que ocorrem no sistema solo-planta-atmosfera. Do total de radiação que chega à superfície, parte é imediatamente refletida, parte é transmitida e outra é absorvida pelas plantas de forma diferenciada, dentro do espectro solar. Segundo Gates (1965) as plantas absorvem cerca de 50\% da radiação de ondas curtas incidente e $97 \%$ da radiação de ondas longas proveniente da atmosfera. A razão entre as radiações de ondas curtas refletidas e incidentes, é denominada coeficiente de reflexão ou albedo, um parâmetro fundamental na determinação do balanço de radiação à superfície. $\mathrm{O}$ albedo de uma superfície vegetada varia ao longo do período diurno, em função do ângulo de elevação do sol e ao longo do ciclo de desenvolvimento da cultura, em função do grau de cobertura vegetal, tipo e estado de umidade do solo, condições de umidade do ar e da quantidade e tipo de cobertura de nuvens (Blad \& Baker, 1972; Leitão, 1989; Azevedo et al., 1990a e b). De acordo com Ayoade (1983) os raios solares verticais geralmente produzem albedo menor que os raios solares oblíquos ou inclinados; desta forma, o albedo de uma superfície é elevado após o nascer e antes do pôr-dosol, enquanto seus menores valores ocorrem em torno do meio dia.

André \& Viswanadham (1983) estudando uma cultura de soja, observaram que o albedo variou com o desenvolvimento desta, porém se mostrou inversamente relacionado com a altura das plantas. Segundo Fritschen (1967) o albedo do algodão tende a aumentar com o desenvolvimento da cultura, variando de 0,18 , com o solo descoberto, a 0,27 quando a cultura atinge $1,15 \mathrm{~m}$ de altura. Alves (1997) verificou que o albedo do meloeiro variou de $0,18 \operatorname{logo}$ após a semeadura a 0,23 no período de máximo desenvolvimento vegetativo da cultura, decrescendo no subperíodo de maturação dos frutos.

$\mathrm{O}$ albedo pode ser influenciado pela irrigação e, como conseqüência, afetar diretamente o balanço de radiação à superfície e, indiretamente, o balanço de energia, o que pode produzir erros signifivativos, principalmente nas estimativas da evapotranspiração. Leitão et al. (1996) estudando o comportamento dos componentes do balanço de radiação em uma cultura de amendoim sob diferentes tratamentos de irrigação, observaram que, em parcelas mais fechadas, houve redução de $1 \%$ no albedo médio diário no dia da irrigação e de $2 \%$ no dia posterior, enquanto em parcelas menos fechadas, ou seja, naquelas com maior exposição do solo, verificaram redução ainda mais acentuada, cerca de 4\%. Azevedo et al. (1990) estudando o balanço de radiação sobre algumas culturas irrigadas no semi-árido do Nordeste do Brasil, observaram que, nos dias anteriores às irrigações, o albedo foi cerca de $3 \%$ superior àquele dos dias posteriores às irrigações. Considerando-se que a irrigação modifica o estado do solo e, como conseqüência, a resposta espectral da superfície, com efeito direto no balanço de radiação e, por conseguinte, no balanço de energia, o presente trabalho teve como objetivo analisar a influência da irrigação sobre o albedo, particularmente durante um dia e ao longo de todo o ciclo de desenvolvimento da cultura de amendoim.

\section{MATERIAL E MÉTODOS}

Este estudo foi conduzido no período de 20 de setembro a 23 de dezembro de 1996, no perímetro irrigado da Estação Experimental da Companhia de Desenvolvimento do Vale do São Francisco - CODEVASF, no município de Rodelas, BA (lat. 0850'S; long. 38 $46^{\circ}$ 'W; alt. $270 \mathrm{~m}$ ). A cultura utilizada foi o amendoim (Arachis hipogaea L.), cultivar BR-1; para monitorar o experimento, três torres micrometeorológicas foram instaladas ao longo do campo experimental, orientadas na direção predominante do vento, distanciadas $25 \mathrm{~m}$ uma da outra (Figura 1) com sensores para medir temperatura do ar, e úmida a 1 e $2 \mathrm{~m}$, radiação solar incidente e refletida, saldo de radiação, fluxo de calor no solo e velocidade do vento a 1 e $2 \mathrm{~m}$ acima do solo. É importante salientar que, tanto o sensor de radiação global como o de radiação refletida, foram instalados de modo que não fossem atingidos pela irrigação, a qual foi efetuada por aspersores com $0,5 \mathrm{~m}$ de altura. $\mathrm{O}$ radiômetro global foi instalado a $2,5 \mathrm{~m}$ de altura e o refletido a $1 \mathrm{~m}$, com proteção especial. A aquisição dos dados foi efetuada através de um MICROLOGGER 21X, a cada segundo, que possibilitou médias a cada 5 min e determinação dos totais diários.

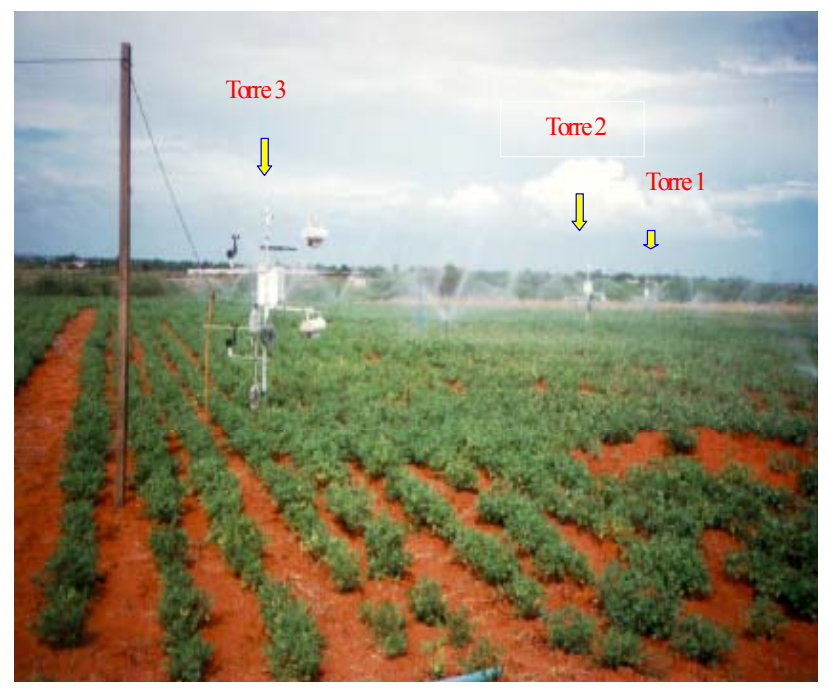

Figura 1. Posição das torres micrometeorológicas no campo experimental

\section{Balanço de radiação}

O balanço de radiação à superfície é dado pela soma dos fluxos de radiação de ondas curtas e ondas longas e pode ser expresso pela seguinte equação:

$$
\mathrm{Rn}=(\mathrm{K} \downarrow-\mathrm{K} \uparrow)+(\mathrm{L} \downarrow-\mathrm{L} \uparrow)
$$

donde:

Rn - saldo de radiação

$\mathrm{K} \downarrow$ - radiação de ondas curtas incidente

$\mathrm{K} \uparrow$ - radiação de ondas curtas refletida pela superfície

$\mathrm{L} \downarrow$ - radiação de ondas longas da atmosfera

$\mathrm{L} \uparrow \quad$ - radiação de ondas longas emitida pela superfície.

Quando são conhecidos o albedo (r) e a temperatura da superfície $\left(\mathrm{T}_{\mathrm{S}}\right)$ a Eq. (1) pode ser escrita na forma: 


$$
\mathrm{Rn}=(1-\mathrm{r}) \mathrm{K} \downarrow+\left(\mathrm{L} \downarrow-\varepsilon \sigma \mathrm{T}_{\mathrm{S}}{ }^{4}\right)
$$

em que:

$$
\begin{array}{ll}
\varepsilon & \text { - emissividade da superfície } \\
\sigma & \text { - constante de Stefan-Boltzmann }\left(5.67 \times 10^{-8} \mathrm{~W} \mathrm{~m}^{-2} \mathrm{~K}^{-4}\right) .
\end{array}
$$

\section{Determinação do albedo (r)}

O albedo instantâneo foi obtido pela razão entre a radiação refletida e a radiação incidente, ambas mediadas a cada cinco minutos. $\mathrm{O}$ albedo médio diário foi determinado pela razão entre os valores médios de radiação refletida e radiação incidente, obtidos a cada cinco minutos e integrados para o período diurno, durante o ciclo de desenvolvimento da cultura. Para tanto, utilizou-se a Eq. (3) para determinação do albedo.

$$
\mathrm{r}=\mathrm{K} \uparrow / \mathrm{K} \downarrow
$$

\section{RESULTADOS E DISCUSSÃO}

Nas Figuras 2A e B é apresentado o comportamento dos componentes do balanço de radiação durante um dia com irrigação e outro sem irrigação. Verifica-se que a ocorrência de irrigação produziu redução imediata na radiação refletida $(\mathrm{K} \uparrow)$ enquanto a radiação global $(\mathrm{K} \downarrow)$ permaneceu inalterada. Fisicamente, o que ocorreu foi uma absorção maior de radiação pela superfície, devido à presença da água no solo, que culminou, conseqüentemente, numa redução do poder de reflexão da
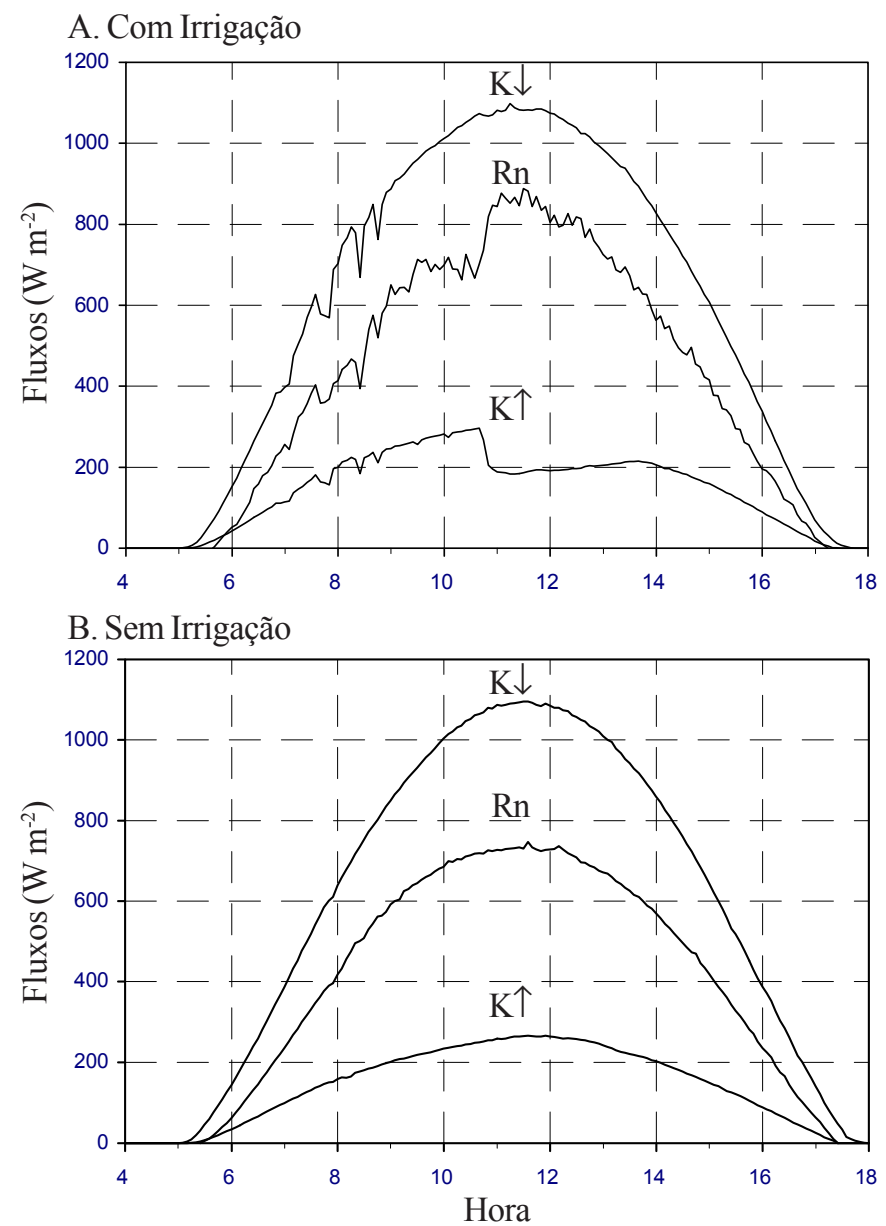

Figura 2. Comportamento dos componentes do balanço de radiação durante um dia - A. com irrigação e B. sem irrigação superfície, resultando num albedo menor, fato comprovado com o aumento da radiação líquida (Rn) ou seja, a medida em que mais radiação é absorvida pela superfície, mais radiação estará disponível ao meio.

Na Figura 3 é apresentado o comportamento médio horário diurno do albedo, para um dia com irrigação e outro sem irrigação. Nota-se que, para o dia sem irrigação, que também foi de céu claro, o albedo apresentou valores mais ou menos simétricos entre o nascer do sol ao meio dia e deste ao pôr-do-sol, com os máximos ocorrendo nas primeiras horas da manhã e final da tarde, enquanto os mínimos ocorreram em torno do meio dia; contudo, no dia em que ocorreu irrigação, a qual teve início por volta de $10: 50 \mathrm{~h}$ e término em torno das $11: 50 \mathrm{~h}$, verificou-se que houve queda brusca no albedo médio entre 10 e $12 \mathrm{~h}$ de cerca de $9 \%$, só voltando para o valor próximo da normalidade no final da tarde, ou seja, devido o solo local ser constituído, em sua maior parte, de areia (cerca de 95\%) e possuir pequena capacidade de retenção de água, apesar dos efeitos da irrigação serem sentidos imediatamente, isto prevalece apenas por algumas horas.

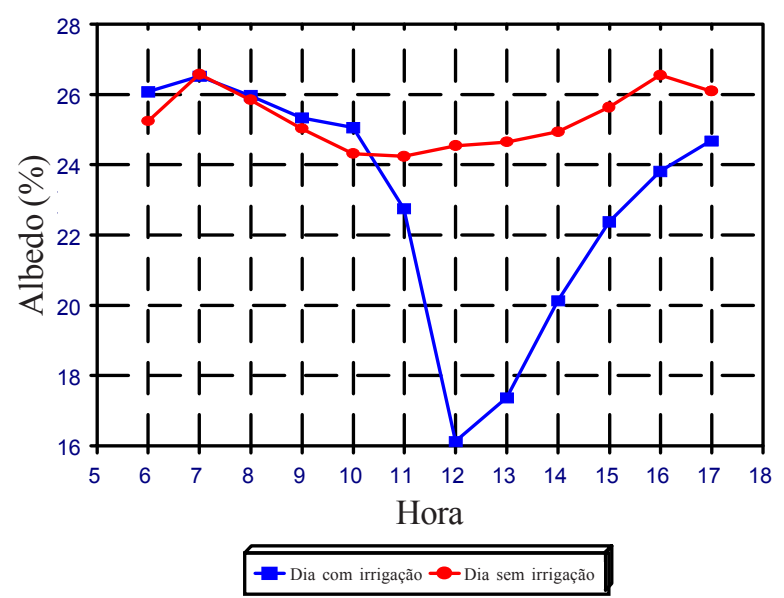

Figura 3. Comportamento médio horário do albedo em dias com e sem irrigação

Na Figura 4 é apresentado o comportamento do albedo médio diário ao longo do ciclo de desenvolvimento da cultura do amendoim, podendo-se observar que na fase inicial do experimento, quando o solo ainda estava totalmente descoberto, devido à composição arenosa da superfície, houve maior reflexão, com o albedo alcançando o valor de $29,8 \%$; contudo, na medida em que a cultura se foi desenvolvendo e passando a cobrir o solo, o albedo foi diminuindo, até atingir seu valor mínimo de $18,6 \%$ no final da fase de desenvolvimento de vagem. Observa-se, também, que o albedo apresentou certas flutuações ao longo do ciclo de desenvolvimento da cultura, especificamente nos dias em que houve irrigação ou ocorrência de precipitação, com queda de até $7,6 \%$ nos dias de irrigação. Uma análise para cada estágio de desenvolvimento indica que o albedo médio, durante o estágio de solo descoberto, foi de $26,7 \%$, no estágio de crescimento e germinação de $25,1 \%$, no estágio de floração e desenvolvimento de vagem $23,7 \%$ e no estágio de maturação de $21,1 \%$. Em termos de valor médio diário para todo o ciclo da cultura registrou-se um albedo de $23,6 \%$.

Considerando-se que o conhecimento do albedo é muito importante para o balanço de radiação e que, quando não se tem medidas de radiação refletida, o mesmo pode ser facilmente 


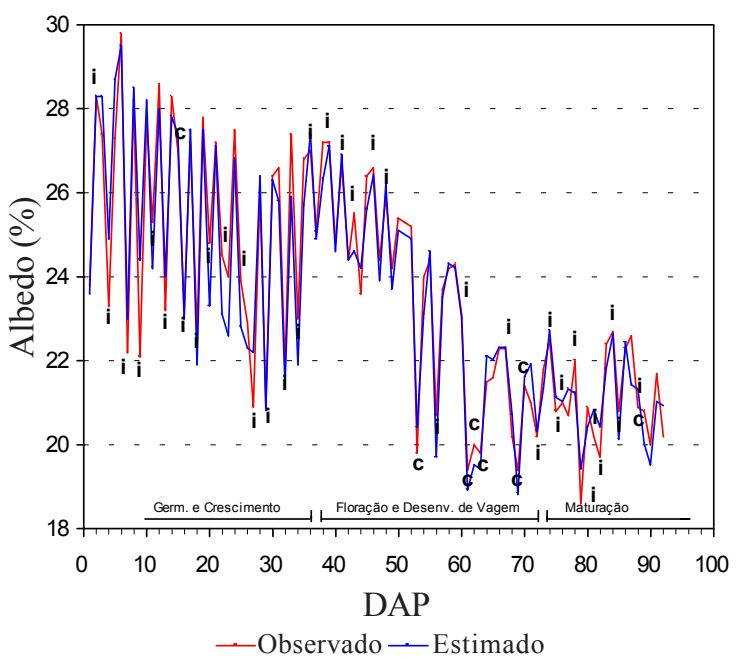

Figura 4. Comportamento do albedo médio diário observado e estimado ao longo do ciclo de desenvolvimento da cultura do amendoim (i - dia com irrigação e c - dia com chuva)

estimado em função do desenvolvimento da cultura procurou-se, com este estudo, obter uma equação que permitisse estimá-lo; para tanto, selecionou-se o albedo médio diário dos dias sem ocorrência de irrigação ou precipitação ao longo do ciclo de desenvolvimento da cultura e, a partir daí, obteve-se, por regressão linear, a seguinte expressão, em função do número de dias após o plantio (DAP): $r=30,1-0,09967 D A P$. Embora esta regressão linear tenha apresentado alto coeficiente de determinação $\left(\mathrm{R}^{2}=0,97\right)$ o seu uso para determinação do albedo em dias com ocorrência de irrigação requer correções, as quais devem ser feitas em função do horário em que as irrigações foram realizadas. Deste modo, a equação anterior, para ser usada também nos dias de irrigação, passa a ter a seguinte forma: $\mathrm{r}=30,1-0,09967 \mathrm{DAP}+\mathrm{C}$, em que $\mathrm{C}$ é o fator de correção em função do horário de irrigação a ser aplicado nos dias de irrigação e no dia seguinte.

Para se comparar o albedo estimado em função do número de dias após o plantio (DAP) com o albedo observado, são apresentadas, na Figura 4, as curvas relativas a ambos os albedos. Analisando-se esta figura, verifica-se que a curva do albedo estimado apresenta razoável aproximação com a curva do albedo observado, indicando boa performance da equação obtida.

$\mathrm{Na}$ Tabela 1 tem-se os valores das correções em função do horário de irrigação a serem aplicadas ao albedo nos dias de irrigação e no dia seguinte. Para o caso de ocorrência de chuva, observou-se que, ao longo da fase experimental, o albedo apresentou redução média de 4,4\% nos dias de ocorrência de chuva e de 1,6\% no dia seguinte. É oportuno lembrar que nos dias de ocorrência de precipitação, ao contrário do que ocorre nos dias de irrigação, tanto a cultura como os sensores de radiação são atingidos pela chuva, particularmente o sensor de radiação global, o que implica em leituras alteradas, que não representam a realidade; por outro lado, é importante observar que estas correções só são válidas para as condições similares às observadas em Rodelas, BA, já que estão diretamente relacionadas às condições climáticas e do solo da região.
Tabela 1. Fatores de correção a serem aplicados às estimativas do albedo, nos dias de irrigação e no dia seguinte, em função do horário de irrigação

\begin{tabular}{ccc}
\hline Horário de & \multicolumn{2}{c}{ Fator de Correção } \\
\cline { 2 - 3 } Irrigação & Dia da Irrigação & Dia Seguinte \\
\hline 06 às $10 \mathrm{~h}$ & $-6,4$ & $-0,7$ \\
10 às $14 \mathrm{~h}$ & $-4,8$ & $-0,9$ \\
14 às $16 \mathrm{~h}$ & $-1,1$ & $-1,5$ \\
Após às $16 \mathrm{~h}$ & $+0,9$ & $-1,5$ \\
\hline
\end{tabular}

Analisando-se os dados da Tabela 1, verifica-se que nos dias em que a irrigação foi efetuada entre 6 e $10 \mathrm{~h}$, ocorreu redução média do albedo de $6,4 \%$, porém no dia seguinte este praticamente volta à normalidade, com redução de apenas $0,7 \%$; já nos dias em que a irrigação se processou entre 10 e 14 h, o albedo apresentou redução média de $4,8 \%$ e, no dia seguinte, de $0,9 \%$. Nos dias em que houve irrigação entre 14 e $16 \mathrm{~h}$, ele mostrou redução média de $1,1 \%$ no dia da irrigação e, no dia seguinte, de $1,5 \%$. Por último, quando a irrigação foi processada após as $16 \mathrm{~h}$, ocorreu aumento de $0,9 \%$ no albedo deste dia e redução de $1,5 \%$ no dia seguinte. Este aumento do albedo está associado ao fato de que a irrigação, sendo efetuada no final da tarde, em horários em que o sol apresenta ângulos de elevação pequenos, contribuiu para uma reflexão bem mais acentuada pois, neste caso, a superfície passa a atuar como uma espécie de espelho, refletindo bem mais radiação.

\section{CONCLUSÕES}

1. O albedo apresentou valor relativamente elevado na fase inicial do experimento $(\mathrm{r}=29,8 \%)$ e, na medida que a cultura se foi desenvolvendo, o mesmo foi diminuindo, até atingir seu valor mínimo no final da fase de desenvolvimento de vagem $(\mathrm{r}=18,6 \%)$.

2. O albedo pode ser estimado com razoável precisão, em função do número de dias após o plantio (DAP) com correção para os dias em que ocorre irrigação.

3. Nos dias em que a irrigação ocorreu pela manhã, esta exerceu influência sobre o albedo médio diário, reduzindo-o em $6,4 \%$.

\section{REFERÊNCIAS BIBLIOGRÁFICAS}

ALVES, A.V. Balanços de radiação e energia em um cultivo de melão. Campina Grande, PB: UFPB, 1997, 95p. Dissertação Mestrado. DCA - CMM TD No 10

ANDRÉ, R.G.B.; VISWANADHAM, Y. Radiation balance of soybeans grown in Brazil. Agricultural Meteorology, Amsterdam, v. 30, p.157-173, 1983.

AYOADE, J.O. Introdução à climatologia para os trópicos. Amsterdam: Ed. Bertrand Brasil S.A. $2^{\mathrm{a}}$ ed., 1983, p.332.

AZEVEDO, P.V.; LEITÃO, M.M.V.B.R.; SOUZA, I.F. Balanço de radiação sobre culturas, nas condições semi-áridas do cariri paraibano. CONGRESSO BRASILEIRO DE METEOROLOGIA, 4, Salvador, BA. Anais... I. 1990a, p. 33-38. AZEVEDO, P.V.; LEITÃO, M.M.V.B.R.; SOUZA, I.F.; MACIEL, G.F. Balanço de radiação sobre culturas irrigadas no semi-árido do Nordeste do Brasil. Revista Brasileira de Meteorologia, Santa Maria, v.5, n.1, p.403-410, 1990b. 
BLAD, B.L.; BAKER, D.G. Reflected radiation from a soybean crop. Agronomy Journal, Madison, v.64, p.277-280, 1972.

FRITSCHEN, L.J. Net and solar radiation relations over irrigated field crops. Agricultural Meteorology, Amsterdam, v.4, p.56-62, 1967.

GATES, D.M. Radiant energy, its receipt and disposal. Agricultural Meteorology (In: Waggonerm, P.E. ed.) Meteorology Monograph. v.6, chap. 1, p.1-26. Boston: American Meteorological Society, 1965.
LEITÃO, M.M.V.B.R. Balanço de radiação e energia numa cultura de soja irrigada. Campina Grande, PB:UFPB. 1989, 110p. Dissertação Mestrado. DCA/CMM - TD nº 03

LEITÃO, M.M.V.B.R.; FIDELES FILHO, J.; SILVA, L.C.; RAO, T.V.R. Comportamento dos componentes do balanço de radiação em amendoim sob diferentes tratamentos de irrigação. CONGRESSO BRASILEIRO DE METEOROLOGIA, 9, Campos do Jordão, SP: Sociedade Brasileira de Meteorologia, Anais... I, 1996, p.417-420. 